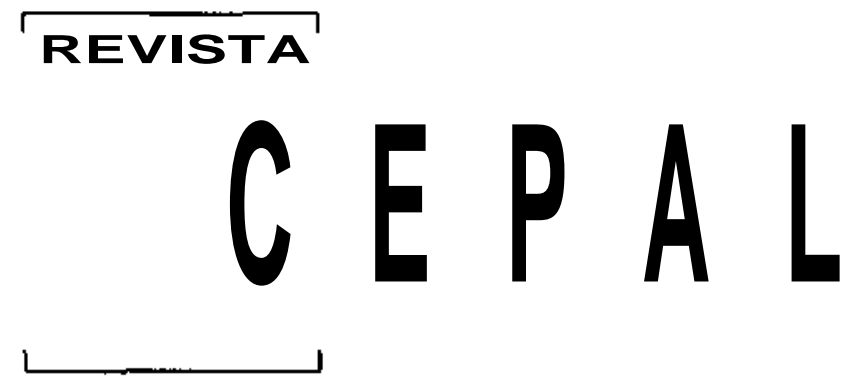

NUMERO 65

AGOSTO 1998 SANTIAGO DE CHILE

ÓSCAR ALTIMIR

Director

EUGENIO LAHERA

Secretario Técnico

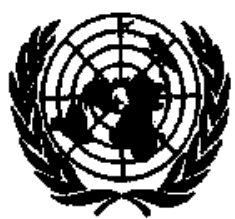

NACIONES UNIDAS 
Distribución del ingreso, pobreza y gasto social en América Latina José Antonio Ocampo

Gasto militar y el desarrollo en América Latina

Eugenio Lahera y Marcelo Ortúzar

Crecimiento, justicia distributiva y política social

Andrés Solimano

Equidad, inversión extranjera y competitividad internacional

Adolfo Figueroa

Tensiones en el ajuste estructural en América Latina: asignación vs. distribución

Daniel M. Schydlowsky

Competitividad y regulaciones laborales

Luis Beccaria y Pedro Galín

Familias latinoamericanas: convergencias y divergencias de modelos y políticas

Irma Amagada

Los acuerdos de libre comercio y el trabajo de las mujeres:

el caso de Chile

Alicia Frohmann y Pilar Romaguera

Evolución macroeconómica del Paraguay 1989-1997:

burbuja de consumo y crisis financiera

Stéphane Straub

Estrategias de las empresas mexicanas en sus procesos de internacionalización

Alejandra Salas-Porras

La regulación de la prestación privada de servicios de agua potable y alcantarillado

Terence R. Lee y Andrei S. Jouravlev

Promoción de la calidad para mejorar la competitividad

Hessel Schuurman

Publicaciones recientes de la CEPAL 


\section{Distribución del ingreso, pobreza y gasto social en América Latina}

José Antonio Ocampo

Secretario Ejecutivo

de la CEPAL
La gran desigualdad social ha sido una característica frustrante

del desarrollo económico latinoamericano. No en vano Améri-

ca Latina se ha caracterizado por ser la región del mundo con los más elevados índices de desigualdad en la distribución del ingreso. Los niveles de pobreza, aunque inferiores a aquellos típicos de otras partes del mundo en desarrollo, siguen siendo extremadamente elevados y, para el conjunto de la región, se encuentran hoy por encima de los niveles que se observaban antes de la crisis de la deuda. Estas son las condiciones que se enfrentan hoy a los nuevos elementos que han alterado la dinámica económica y social de la región. Entre ellos cabe mencionar cuatro: las reformas estructurales emprendidas en todos los países, el proceso de globalización que las ha acompañado, la recuperación de) crecimiento económico y las nuevas reformas iniciadas en el frente del gasto social y de los servicios sociales, como parte de las llamadas reformas de "segunda generación". Este artículo plantea algunas hipótesis sobre los efectos de estos nuevos acontecimientos sobre la pobreza y la desigualdad y analiza sus implicaciones para la política social. 


\section{Pobreza, desigualdad y sus determinantes}

La "década perdida" fue un período de marcado deterioro en materia de pobreza en América Latina. La región retrocedió en este terreno, en efecto, y en 1990 sus niveles de pobreza eran superiores incluso a los existentes a comienzos de los años setenta. En los noventa, por el contrario, la recuperación del crecimiento económico ha impulsado una importante mejoría en esos indicadores, aunque el promedio regional se encuentra aún por encima de los niveles prevalecientes antes de la crisis. De este modo, mientras en 1980 el 35\% de los hogares se encontraba en situación de pobreza, y en 1990 dicha proporción se ubicaba en el 41\%, en 1994 se mantenía en el 39\% (gráfico 1). En términos de distribución del ingreso) la década de los años ochenta fue también de deterioro. La expectativa de que la renovación del crecimiento económico revertiría dicha tendencia no se ha materializado, de manera que los niveles de desigualdad se encuentran hoy por encima de los ya elevados que existían antes de la crisis de la deuda (BID, 1997; CEPAL, 1997).

Estas tendencias globales esconden, como es obvio, patrones heterogéneos en los distintos países de la región. Según los estudios comparativos existentes, en sólo uno de ellos - Uruguay - tanto los niveles de pobreza como los de equidad han mejorado en relación con los que se observaban a comienzos de los años ochenta. En varios otros - Brasil, Panamá y, de acuerdo con algunos estudios, Colombia- los niveles de pobreza han bajado, pero no han mejorado los de equi-

GRAFICO II

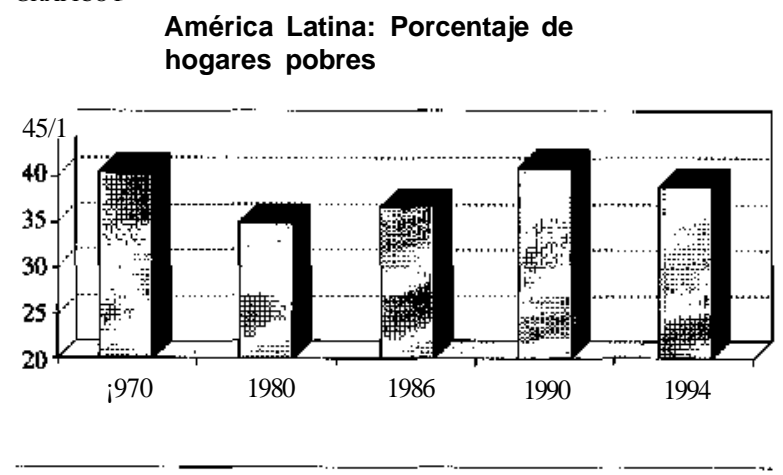

I I Presentación en la primera Conferencia de las Américas convocada por la Organización de los Estados Americanos, Washington, 6 de marzo de 1998. dad. El caso chileno es más complejo: la pobreza se ha reducido notablemente en relación con los niveles de mediados de los años ochenta y quizás con los de comienzos de dicha década (para los cuales no existen estimaciones), pero apenas ha regresado a los de comienzos de los años setenta; ${ }^{1}$ en tanto que la desigualdad en la distribución del ingreso es superior a la de entonces y ha sido renuente a disminuir durante el período reciente de fuerte reducción de la pobreza.

La explicación de estas tendencias ha dado lugar a una importante controversia sobre los efectos del comportamiento macroeconómico, de las reformas estructurales y de la globalización sobre los indicadores , sociales. A partir del ensayo pionero de Morley (1994), diversos trabajos han confirmado que la pobreza tiende a reducirse con el crecimiento económico, lo que explicaría, por lo tanto, el comportamiento favorable que ha tenido dicha variable con el mayor crecimiento que ha acompañado el proceso de reformas. Por el contrario, existe un creciente cuerpo de estudios que indica que la liberalización económica y la globalización han tendido a deteriorar la distribución del ingreso.

Uno de los autores que ha venido aseverando esto con mayor énfasis en los últimos años es Albert Berry, Este autor ha mostrado en varios trabajos que existe información en el último cuarto de siglo según la cual la aplicación de medidas de liberalización económica ha estado asociada con deterioros, a veces considerables, en la distribución del ingreso (por ejemplo, véase Berry, 1997). Esta información proviene, según el autor, de las experiencias de Argentina, Chile, Colombia, México, la República Dominicana y Uruguay, en tanto que Costa Rica es una excepción. El trabajo comparativo de Robbins (1996) indica también que los procesos de apertura comercial tuvieron efectos desfavorables sobre la equidad en varios países de la región.

\footnotetext{
1 Aunque existen problemas de comparabilidad en las cifras, CEPAL (1990) estimó que la proporción de hogares pobres en Chile era de $38.1 \%$ en 1987 , contra $17 \%$ en 1970 , y que el porcentaje de personas pobres era de 44.4 contra $20 \%$ en dichos años. Las revisiones posteriores elevaron ligeramente las estimaciones de 1987 al 39,1 y 45.1\%. Los cálculos más recientes, correspondientes a 1996, indican que el porcentaje de hogares pobres y personas pobres se ha reducido al 19.7 y $23.3 \%$, respectivamente.
} 
En trabajos recientes de la CEPAL (por ejemplo, en CEPAALhecho casi universal, ya que ha afectado a varios 1997) se indica que las principales presiones adversas sobre la distribución del ingreso provienen, en la década actual, del aumento de las disparidades entre las retribuciones de los trabajadores más calificados y las de los menos calificados, en condiciones de escasa generación de empleos calificados y de mayor desigualdad intrasectorial e intersectorial del producto por persona ocupada. En efecto, la diferencia de remuneraciones aumentó en forma casi generalizada en los países de la región durante el primer lustro de los años noventa (gráfico 2). Los estudios mencionados, al igual que los de la OIT (véase OIT, 1997), indican que en la región el empleo ha crecido menos que la población económicamente activa y, en especial, que los nuevos puestos de trabajo se han concentrado en el sector informal. De acuerdo con las estimaciones de la OIT, ocho de cada diez puestos de trabajo creados en los años noventa corresponden a ocupaciones de baja calidad en el sector informal.

La creciente desigualdad de las remuneraciones según los niveles de calificación de la mano de obra no sólo ha sido una característica de los procesos de reestructuración próductiva en la región. De hecho, un reciente informe (UNCTAD, 1997) indica que puede ser

GRAFICO 2

América Latina: Diferencias de remuneraciones laborales, 1990 y 1994

A. Profesionales con respecto a trabajadores formales

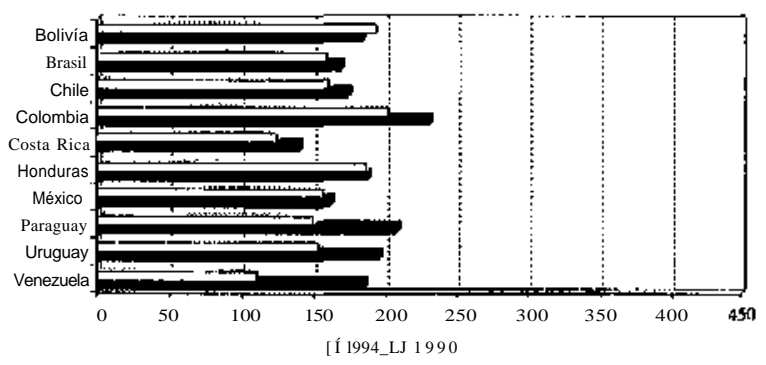

B. Profesionales con respecto a trabajadores informales

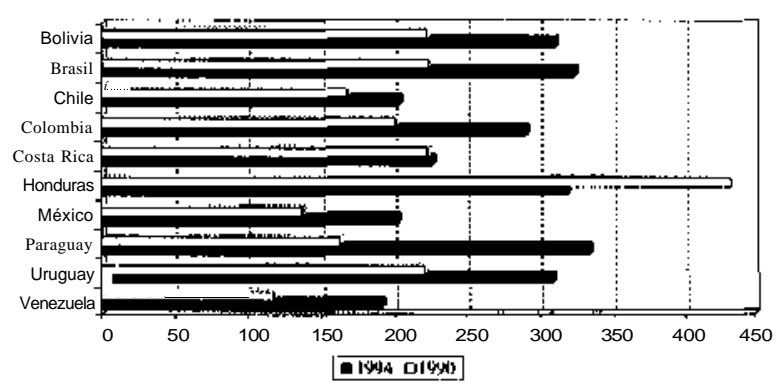

Fuente: CEPAL, 1997. países industrializados y a algunas economías de rápido crecimiento en el Asia-Pacífico y ha generado, en particular, una presión severa sobre las clases medias de muchos países.

] Existen varias explicaciones posibles para estas tendencias. La más sugestiva es la de Rodrik (1997), ' según la cual la globalización acentúa la asimetría entre los factores que pueden cruzar con mayor facilidad las fronteras nacionales —el capital y la mano de obra más calificada - y aquellos que no pueden hacerlo - la mano de obra menos calificada- La posibilidad de relocalizar la producción hace que la demanda laboral se torne más elástica en todos los países, reduciendo la capacidad de negociación de los trabajadores y aumentando la inestabilidad de sus ingresos frente a perturbaciones de la demanda.

Otros autores han sugerido explicaciones distintas. Según Berry (1997), existen importantes economías de escala en el comercio y el financiamiento internacionales, que se reflejan en la mayor participación en estas actividades de las firmas más grandes dentro de cada sector, que a su vez son las que hacen uso más intensivo de capital y/o mano de obra más calificada. Esto explicaría por qué un crecimiento relativo en las actividades más asociadas al comercio internacional podría traducirse en una mayor desigualdad. Se ha sugerido también que las mayores transferencias de tecnología que genera el propio comercio, incluidas las que se transmiten a través de las importaciones de maquinaria y equipo, pueden llevar a países en desarrollo inmersos en procesos de internacionalización, a adoptar tecnologías que hacen uso intensivo de mano de obra calificada, diseñadas en función de las necesidades de los países industrializados (Robbins, 1996).

En el caso latinoamericano, puede haber explicaciones complementarias de este fenómeno. Una de ellas es que el proceso de liberalización comercial tuvo lugar después de una década de debilitamiento del gasto social. El sesgo de la demanda laboral hacia mano de obra más calificada enfrentó, por lo tanto, una oferta inelástica de dichos trabajadores. No hubo, además, durante el proceso mismo de liberalización, esfuerzos claros para acoplar la demanda y la oferta de calificaciones. Otra explicación señalaría que algunos elementos de la política macroeconómica que acompañaron el proceso de reformas, en especial la tendencia a la revaluación de los tipos de cambio y la apertura de la cuenta de capitales, generaron patrones de crecimiento en los cuales las exportaciones mostraron un menor dinamismo que las importaciones, y los sectores pro- 
ductores de bienes transables menor dinamismo que los de bienes y servicios no comercializables internacionalmente, generando sesgos en la demanda de mano de obra que se reflejaron en el comportamiento relativo de las remuneraciones. ${ }^{2}$ El manejo macroeconómico se ha caracterizado, además, por fuertes ciclos de "pare $y$ siga" que, en conjunto con las fluctuaciones de los flujos de capital, han mantenido una alta volatilidad de los ritmos de crecimiento; dificultando así la generación de puestos de trabajo más estables.

Las consideraciones anteriores no pretenden en modo alguno mostrar que las reformas económicas son la causa de los actuales niveles de desigualdad social en la región. Lejos de ello. Según lo señalamos al comienzo de este artículo, la desigualdad tiene en Amé- rica Latina raíces muy profundas. Está asociada muy especialmente a la gran desigualdad en la distribución del capital humano y de la riqueza. ${ }^{3}$ En muchos países, además, la etapa de sustitución de importaciones se caracterizó por un deterioro distributivo y la experiencia de los años ochenta puede considerarse una demostración fehaciente de los costos sociales asociados tanto a los desequilibrios macroeconómicos (por ejemplo, de los efectos regresivos de la desestabilización del nivel general de precios) como al impacto inicial de los procesos de ajuste orientados a corregirlos. Sin embargo, dichas consideraciones indican que, pese a tener efectos positivos sobre el crecimiento, la liberalización económica y la globalización, lejos de reducir, han aumentado el reto de la equidad.

\section{II}

\section{Gasto público social y equidad}

Según se desprende de la sección anterior, uno de los grandes desafíos de América Latina consiste en demostrar que el nuevo modelo de desarrollo es compatible con la corrección gradual de las grandes desigualdades sociales existentes. De no lograrse este objetivo, las bases políticas de las reformas —que han sido sólidas hasta ahora, en gran medida porque el retorno a la estabilidad macroeconómica ha sido visto como positivo por el conjunto de la población- podrían deteriorarse. Lo que es igualmente grave, tal vez se generarían tensiones sociales que podrían afectar la gobernabilidad y erosionar las bases de los consensos políticos que han permitido el fortalecimiento de la democracia en la región, sin duda otro de los grandes logros de los últimos años.

La experiencia internacional muestra resultados sorprendentes sobre el manejo de los riesgos sociales que implica la globalización. El estudio de Rodrik (1997), ya citado, indica que la mayor apertura de las economías ha estado compensada en el pasado por

\footnotetext{
2 Ya sea como resultado de la apertura comercial como tal o de los incentivos generados por las políticas macroeconómicas, o de ambos, se han debilitado también los encadenamientos de los sectores exportadores con las economías internas, cuyos efectos sobre la generación de empleo deberían también explorarse. Debe resaltarse, sin embargo, que como consecuencia del auge del comercio intrarregional, parte de estos menores encadenamientos nacionales han sido sustituidos por crecientes encadenamientos regionales.
}

mayor protección social del Estado a la población, lo que se ha reflejado en una relación positiva entre el grado de apertura y el tamaño del Estado. Esta apreciación, según dicho autor, es válida tanto para la Organización de Cooperación y Desarrollo Económicos (OCDE) como para una muestra amplia de 115 países. Esto parecería indicar que la tensión distributiva generada por los procesos de apertura se ha abordado hasta ahora intercambiando liberalización económica por mayor protección del Estado a través de una política social más activa.

El gasto social es, sin duda, el componente más importante a través del cual el Estado incide sobre la distribución del ingreso. Hay, en efecto, muchas pruebas de que el efecto del gasto social sobre la distribución secundaria del ingreso es significativo. Además, a largo plazo, la mayor inversión en capital humano permite incidir sobre uno de los determinantes estructurales de la distribución del ingreso. Los estudios existentes muestran que una mayor asignación de recursos a la educación, que permita mejorar la distribución de capital humano en la sociedad, puede tener efectos sobre la distribución del ingreso muy superiores a los que se han estimado en los estudios de incidencia distri-

\footnotetext{
3 Véase al respecto el interesante trabajo de Birdsall y Londoño, 1997.
} 
butiva de corto plazo (véase por ejemplo BID, 1997, pp, 82-83). Como nota de cautela, cabe resaltar que hay también pruebas de que un gran esfuerzo en materia de educación tenderá a traducirse en rentabilidades decrecientes de la inversión en ella, por lo cual sus consecuencias redistributivas (aunque no aquellas sobre el crecimiento) pueden estar algo sobreestimadas. Quizás puedan lograrse también efectos importantes si los esfuerzos se orientan a mejorar la distribución de activos, pero ésta es un área muy poco explorada que debe recibir una mayor atención, y que incluye la redistribución de activos sin generar distorsiones en la actividad económica (como los avances institucionales para canalizar crédito a pequeñas empresas o microempresas sin alterar el mercado crediticio, o los nuevos esquemas de reforma agraria que utilizan activamente el mercado de tierras).

Los datos disponibles para América Latina sobre la distribución secundaria del ingreso indican que, en términos absolutos, los sectores de mayores ingresos se benefician más del gasto social. Sin embargo, como proporción de los ingresos de cada estrato, los subsidios que se canalizan a través de dicho gasto son mayores para los sectores más pobres de la población. Este patrón global es el resultado de los impactos distributivos muy diferentes que tienen los distintos tipos de gastos. La focalización hacia los pobres (es decir, la proporción del gasto que se destina a los pobres en relación con la proporción de la población en estado de pobreza) es elevada cuando se trata de gastos en salud, educación primaria y, en menor medida, educación secundaria (gráfico 3). Por el contrario, los gas-

GRAFICO 3

América Latina (10 países): Focalización del gasto social en los pobres ${ }^{a}$

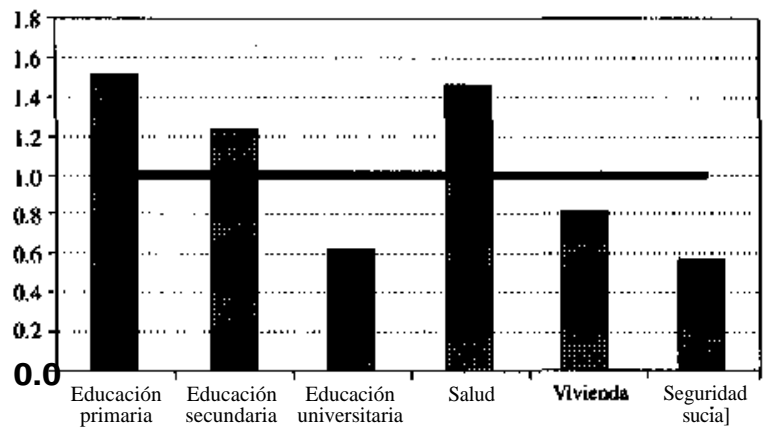

Fuente: CEPAL, 1998

a Los datos presentados se refieren al índice promedio de focalizaciôn de diez países para los cuales existe información. En el caso de algunos programas, la información no cubre la totalidad de estos países. tos en seguridad social y en educación superior tienen una tendencia generalmente regresiva. Los gastos en vivienda se encuentran en una situación intermedia, ya que benefician especialmente a estratos medios de la distribución del ingreso.

Estos resultados indican que existe un amplio margen para influir positivamente en la distribución del ingreso a través del gasto social, pero también para mejorar la focalización de éste, como lo señala un reciente estudio de la CEPAL. La forma como se financia no es, por supuesto, irrelevante: un financiamiento con impuestos directos tiende a ser más progresivo que un financiamiento con impuestos indirectos $y$, lo que es igualmente importante, un financiamiento inadecuado, que se traduzca en una aceleración inflacionaria, puede derrotar sus efectos redistributivos favorables (CEPAL, 1998, cap. VI).

La evolución del gasto social en América Latina, según este mismo estudio (véase también CEPAL, 1997), se resume en el gráfico 4. La década de los ochenta se caracterizó por un colapso de la inversión social. Debido al doble efecto de la menor proporción del PIB destinada al gasto social y de la disminución del ingreso por habitante, el gasto social real per cápita se redujo en un $24 \%$. En los años noventa, uno y otro factor han operado en el sentido positivo, por lo cual en 1995 el gasto social per cápita superaba ya en un $18 \%$ los niveles reales prevalecientes antes de la crisis. Este escenario positivo debe matizarse, sin embargo, de dos maneras diferentes. En primer término, sigue habiendo grandes disparidades entre países de la región en la prioridad otorgada al gasto social, con lo cual en muchos de ellos el gasto social es todavía cuantitativamente insatisfactorio. En segundo lugar, una proporción muy elevada del crecimiento del gasto social, especialmente en los países donde éste es alto, se ha

GRÁFICO 4

América Latina: Gasto social, 1981-1995

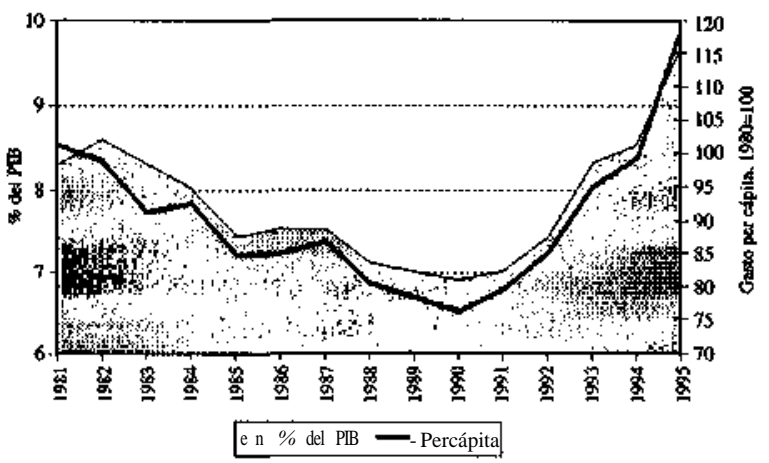

Fuente: CEPAL, 1998 
destinado a seguridad social (y, más específicamente, a pago de pensiones), el componente que está distribuido de manera menos progresiva. Esto significa que el incremento de los gastos en capital humano ha sido menor que el indicado por las cifras mencionadas. En el caso de la educación, el gasto real per cápita para el conjunto de la región apenas había retornado en 1995 a los montos de 1980 y se encontraba todavía por debajo de dicho nivel en un conjunto amplio de países.

Como se desprende de las consideraciones anteriores, existe un margen importante para conjugar las políticas de liberalización económica con una política social más activa, como parecen reflejarlo, por lo demás, los patrones internacionales tradicionales. Esto exige reorientar el gasto público hacia el sector social y focalizar este gasto para maximizar sus efectos distributivos favorables. Algunos países pueden también optar por una expansión adicional del gasto social financiada con mayores ingresos tributarios. Esto fue, de hecho, lo que hizo el gobierno chileno a comienzos de los años noventa, sin perjudicar con ello la inversión real ni el crecimiento económico. En este caso, el impacto distributivo neto dependerá de la forma de financiamiento.

\section{III}

\section{La política social y las reformas de segunda generación}

Los esfuerzos por aumentar y focalizar el gasto social en la región deben complementarse con una importante reorganización del sector que haga más eficiente y eficaz la oferta de servicios sociales. Este es uno de los temas centrales de las llamadas reformas de segunda generación, cuyo propósito esencial es mejorar la eficiencia con la cual operan los mercados e introducir criterios de racionalidad microeconómica en la provisión de aquellos servicios que no los han tenido.

Los debates sobre los servicios sociales han estado orientados a introducir la competencia en su provisión (creación de cuasimercados), incluyendo la participación de agentes privados, y cambios en las modalidades de apoyo estatal (desde los tradicionales subsidios a la oferta hacia subsidios a la demanda). En forma paralela y complementaria, se ha descentralizado la prestación de aquellos servicios que continúan a cargo del Estado, se han creado nuevos esquemas de gestión pública orientados a lograr resultados, se ha dado autonomía efectiva a las entidades públicas prestadoras de servicios y se han establecido mecanismos de participación ciudadana para el control de la gestión pública. Unos y otros componentes de esta reorganización, pero especialmente los primeros, están orientados a enfrentar las "fallas del gobierno" que se han hecho evidentes en el pasado en la provisión de servicios sociales y que se traducen en ineficiencias y baja calidad de los servicios prestados por el Estado.

La experiencia muestra que el sector privado responde en forma dinámica a los incentivos que se le proporcionan. Sin embargo, también indica que su participación está sujeta igualmente a "fallas de mercado", tanto de carácter tradicional, relacionadas con economías de escala, como aquellas asociadas a problemas de información, que dan lugar a fenómenos de competencia imperfecta, selección adversa y riesgos morales (Ocampo, 1996).

En economías con fuertes disparidades distributivas, el problema más importante es la tendencia natural que genera el mercado a que el sector privado oriente su oferta — en cantidad y especialmente en calidad - hacia los sectores de mayores ingresos. Este problema no se soluciona necesariamente con un esquema de subsidios a la demanda y exige, por lo tanto, que el Estado diseñe instrumentos para aumentar la oferta dirigida a los sectores de bajos ingresos. Como la oferta pública ha sido en muchos casos igualmente incapaz de llegar a ciertos sectores de la población (por ejemplo, en los programas de vivienda popular), es preciso promover nuevos agentes, que generalmente tienen un carácter solidario o comunitario.

Las dificultades anteriores se acentúan cuando los esquemas de participación privada no incluyen claros principios de solidaridad, como lo ilustra el sistema de salud en Chile. En este caso, la selección adversa - tanto por estratos socioeconómicos como por los riesgos de salud asociados a la edad de la población cubierta- puede ser muy marcada. Sin embargo, la introducción de elementos de solidaridad no soluciona automáticamente el problema. Así, por ejemplo, para 
evitar las dificultades señaladas, en la reforma del sistema de salud en Colombia se establecieron beneficios totalmente independientes del monto de la cotización, pero la respuesta privada dirigida a los estratos más pobres ha sido igualmente frustrante durante su fase inicial.

La respuesta privada puede ser, además, geográficamente desequilibrada: responde mejor en ciudades grandes, pero es insuficiente en pequeñas poblaciones o en el sector rural, donde -en razón de mínimas economías de escala- puede haber "monopolios naturales" en muchos servicios, en condiciones en las cuales además su provisión no es rentable.

Cuando la oferta responde inadecuadamente - en términos de cantidad o calidad- a los incentivos generados por los subsidios a la demanda, puede ser aconsejable diseñar fórmulas intermedias, que se pueden denominar "subsidios a la oferta con criterios de demanda"; éstos permiten actuar sobre la oferta, incluso en la calidad de los servicios, y obtener al mismo tiempo los beneficios tradicionales de los esquemas de subsidio a la demanda en términos de focalización de los beneficiarios. Estos esquemas consisten en la contratación de servicios con agentes seleccionados para atender a un grupo específico de la población, a través de un sistema de concurso o de la promoción de organizaciones comunitarias o solidarias con el propósito explícito de otorgarles la administración de los servicios correspondientes. Este puede ser también el esquema apropiado para promover la creación de nuevos servicios o mejorar la calidad de los existentes (por ejemplo, para elevar la calidad del sistema educativo, como viene haciéndose en Chile).

Por otra parte, los problemas de información son mucho más graves en los mercados de servicios que en los de bienes. En particular, hay insalvables asimetrías en la información con que cuentan quienes prestan servicios muy especializados y quienes los reciben —entre médico y paciente, en particular, en el caso de la salud-, pero fenómenos similares están igualmente presentes en la educación. Por este motivo, el desarrollo de cuasimercados para la prestación de servicios sociales exige establecer esquemas mínimos de información e instrumentos muy desarrollados de protección a los usuarios.

La participación privada y la creación de cuasi mercados abren, por lo tanto, buenas oportunidades de romper viejos problemas asociados a la provisión pública de servicios sociales, pero no son una panacea. Los problemas mencionados y los conexos al desarrollo de las instituciones necesarias para superarlos no deben minimizarse. Por lo tanto, las reformas que se adopten en este marco deben ser pragmáticas e incorporar un importante componente de gradualismo y aprendizaje por experiencia. Más aún, debe tenerse presente que los nuevos esquemas de participación privada no son en todos los sectores un sustituto de la oferta pública de servicios. Por este motivo, puede ser conveniente diseñar sistemas mixtos, en los cuales operen agentes públicos y privados, en competencia. Sin embargo, en muchos casos la competencia no es viable. Por lo tanto, los esfuerzos por mejorar la oferta pública, a través de la descentralización, la gestión pública orientada a resultados, la autonomía de las entidades prestadoras de servicios y el control ciudadano son elementos esenciales - y centrales - de cualquier reforma en materia de servicios sociales.

Por último, conviene resaltar que uno de los grandes objetivos de las reformas en los servicios sociales debe ser el diseño de sistemas apropiados de regulación, información y control de calidad de los servicios prestados. Esto es particularmente importante cuando, por las características especializadas de los servicios, no está garantizada la transparencia de la información que necesitarían los consumidores para elegir sus proveedores. Este es un tema todavía incipiente, al cual deberán orientarse esfuerzos considerables en los próximos años.

Así las cosas, la eficiencia microeconómica debe ser una guía importante de las reformas de segunda generación, pero también debe serlo la equidad. En la política social, estas reformas deben ser pragmáticas y polifacéticas. Deben aprovechar las oportunidades que brinda una mayor participación de entidades privadas en la prestación de los servicios sociales, pero también aquellas que ofrecen entidades públicas bien administradas, sujetas a competencia, cuando ello es viable. Más aún, deben dirigirse a resolver las deficiencias comunes a unas y otras, entre las cuales destacan la dificultad para llegar con servicios a los sectores más pobres de la población y la falta de sistemas apropiados de información y control sobre la calidad de los servicios prestados. 


\section{Bibliografía}

Berry, A. (1997): Chapter 1: The income distribution threat in Latín America, A. Berry (ed.), Economic Reforms, Poverty, and Income Distribution in Latín America, en prensa.

BID (Banco Interamericano de Desarrollo) (1997): Progreso económico y social en América Latina. Informe 1997, Washington, D.C.

Birdsall, N. y J. L. Londoño (1997): Asset Inequalily does Malter Lessonsfrom Latín America, Working paper, $\mathrm{N}^{\circ} 344$, Washington, D, C, BID.

CEPAL (Comisión Económica para América Latina y el Caribe) (1990): Una estimación de la magnitud de la pobreza en Chile. 1987, LC/L.599, Santiago de Chile.

(1997): La brecha de la equidad, América Latina, el Caribe y la cumbre social, LC/G.1954/Rev.1-P, Santiago de Chile. Publicación de las Naciones Unidas, $\mathrm{N}^{\circ}$ de venta S.97.II.G.11.

(1998): El pacto fiscal Santiago de Chile.
Morley, S. A. (1994): Poverty and Inequality in Latín America: Past Evidence, Future Prospecta, Policy essay, $\mathrm{N}^{\circ} 13$, Washington D.C, Consejo de Desarrollo de Ultramar.

Ocampo, J. A. (1996): Participación privada en la provisión de servicios sociales: el caso colombiano, Coyuntura social, $\mathrm{N}^{\circ}$ 14, Santafé de Bogotá, Tercer Mundo Editores, mayo .

OIT (Organización Internacional del Trabajo) (1997): Panorama laboral '97, Ginebra.

Robbins, D. (1996): Evidence on Trade and Wages in the Developing World, Technical paper, $\mathrm{N}^{\circ} 119$, París, Organización de Cooperación y Desarrollo Económicos (OCDE), Centro de Desarrollo.

Rodrik, D, (1997): Has Globalizatión Gane Too Far?, Washington, D.C, Institute for International Economics (HE).

UNCTAD (Conferencia de las Naciones Unidas sobre Comercio y Desarrollo) (1997): Informe sobre el comercio y el desarrollo 1997, Nueva York. 